\title{
STUDIES OF PERMITTIVITY IN THE TRIGLICINIUM SULPHATE CRYSTALS DOPED WITH CHROMIUM IONS AS A FUNCTION OF TEMPERATURE AND ELECTRIC FIELD
}

\section{J. DzIEDzIC}

Institute of Physics, Technical University of Wrocław

Wybrzeże Wyspiańskiego 27, 53-370 Wrocław, Poland

(Received April 19, 2000; revised version July 7, 2000)

\begin{abstract}
Permittivity in the triglicinium sulphate crystals doped with chromium ions $\mathrm{Cr}^{3+}$ as a function of temperature and of intensity of electric field polarising the crystal was investigated. On the base of thermodynamical theory, the relationship among permittivity, temperature and electric field intensity was calculated. Coefficients of the expansion of the thermodynamic potential were determined. Experimental results were compared with those resulting from the thermodynamical theory.
\end{abstract}

PACS numbers: $64.70 . \mathrm{Kb}$

\section{Introduction}

The triglicinium sulphate (TGS) crystals doped with the $\mathrm{Cr}^{3+}$ chromium ions are characterised by a remarkable change of the elastic properties in comparison to those of the pure crystals [1]. The author of paper [2] considers the doped crystals as the two-phase systems. One phase consists of the non-disturbed crystal lattice and the other is composed of the spherical regions disturbed by the aminopyridine (APd) impurities. Some theories on the scattering of longitudinal ultrasonic wave on the non-interacting impurity centres were presented in papers $[2-6]$.

The authors of paper [7] found the distinct influence of electric field on dielectric properties of the TGS crystals. They found also the displacement of the Curie temperature in a function of electric field.

There have been no complete results available of the studies on the influence of the electric field intensity for the behaviour of the permittivity in crystals doped with chromium ions $\mathrm{Cr}^{3+}$. 


\section{Thermodynamical relations}

The Gibbs potential $(G)$ may be assumed in the following form:

$$
G(T, P)=G_{0}(T)+\frac{1}{2} A P^{2}+\frac{1}{4} B P^{4}-E P,
$$

where $A, B-$ coefficients of the expansion of the thermodynamic potential, $P$ - polarisation, $E$ - electric field intensity, $T$ - temperature. At the thermodynamic equilibrium a polarisation derivative of the potential is equal to zero

$$
\frac{\partial G}{\partial P}=A P+B P^{3}-E=0,
$$

while $A$ may be expressed as

$$
A=\frac{T-T_{\mathrm{C}}}{\varepsilon_{0} C},
$$

where $T_{\mathrm{C}}-$ Curie temperature, $C-$ Curie-Weiss constant, $\varepsilon_{0}-$ permittivity of free space. So that:

$$
E=A P+B P^{3} \text {. }
$$

\subsection{Permittivity}

The electric susceptibility may be given as:

$$
\frac{1}{\lambda}=\frac{\partial E}{\partial P}=A+3 B P^{2}
$$

and the permittivity as:

$$
\varepsilon=\frac{1}{A+3 B P^{2}} \text {. }
$$

The permittivity depends on the electric field intensity producing the polarisation, the value that may be obtained by solving the equation:

$$
P^{3}+\frac{A}{B} P-\frac{E}{B}=0 \text {. }
$$

Let us have

$$
3 p=\frac{A}{B}, \quad 2 q=\frac{E}{B},
$$

then we have:

$$
P^{3}+p P+q=0 \text {. }
$$

This is a third degree equation vs. polarisation. The solution of the equation depends on a sign of a discriminant $\Delta$ :

$$
\Delta=q^{2}+p^{3}=E^{2}+\frac{4}{27} \frac{A^{3}}{B} \text {. }
$$

$\mathbf{1}^{\circ}$ For $\Delta=0$ :

$E^{2}=-\frac{4 A^{3}}{27 B}$. 
Taking $E_{M}=\sqrt{-\frac{4 A^{3}}{27 B}}$ in the ferroelectric phase for $T<T_{\mathrm{C}}$ and $p<0$ for $|E| \leq\left|E_{M}\right|$ :

$$
\begin{aligned}
& P_{1}=2 \sqrt{\frac{-A}{3 B}} \cos \left(\frac{\varphi}{3}\right), \quad P_{2}=-2 \sqrt{\frac{-A}{3 B}} \cos \left(\frac{\pi}{3}-\frac{\varphi}{3}\right), \\
& P_{3}=-2 \sqrt{\frac{-A}{3 B}} \cos \left(\frac{\pi}{3}+\frac{\varphi}{3}\right),
\end{aligned}
$$

where

$$
\varphi=\arccos \left(-\frac{3 E}{2 A} \sqrt{\frac{A}{3 B}}\right), \quad A=-\frac{2\left(T-T_{\mathrm{C}}\right)}{\varepsilon_{0} C}
$$

and

$$
\varepsilon=\frac{1}{A+3 B P^{2}}
$$

$2^{\circ}$ For $T<T_{\mathrm{C}}$ and $|E| \geq\left|E_{M}\right|$ the ferroelectric phase:

$$
A=-\frac{2\left(T-T_{\mathrm{C}}\right)}{\varepsilon_{0} C}, \quad d=\sqrt{\frac{E^{2}}{4 B^{2}}+\frac{A^{3}}{27 B^{3}}}
$$

and

$$
\varepsilon=\frac{1}{A+3 B P^{2}}
$$

where

$$
P_{2}=\sqrt[3]{\frac{E}{2 B}+d}+\sqrt[3]{\frac{E}{2 B}-d}
$$

$3^{\circ}$ For $T \geq T_{\mathrm{C}}, p>0$ and $E \neq 0$ the paraelectric phase:

$$
A=\frac{\left(T-T_{\mathrm{C}}\right)}{\varepsilon_{0} C}, \quad d=\sqrt{\frac{E^{2}}{4 B^{2}}+\frac{A^{3}}{27 B^{3}}}
$$

and

$$
\varepsilon=\frac{1}{A+3 B P^{2}}
$$

where

$$
P_{3}=\sqrt[3]{\frac{E}{2 B}+d}+\sqrt[3]{\frac{E}{2 B}-d}
$$

On the base of the experimental results, the phase transition temperature, the coefficients of the expansion in a series of thermodynamic potential and the Curie-Weiss constant were estimated for the TGS crystals doped with the $\mathrm{Cr}^{3+}$ chromium ions.

Using the thermodynamical relations and the values of constants obtained for the TGS crystals doped with the $\mathrm{Cr}^{3+}$ ions as $B=6.5 \times 10^{11}\left[\mathrm{~V} \mathrm{~m} \mathrm{~m}^{5} / \mathrm{C}^{3}\right]$, $T_{\mathrm{C}}=326.3[\mathrm{~K}], C=3560[\mathrm{~K} \mathrm{C} /(\mathrm{F} \mathrm{V})]$, the dependences of the permittivity $\varepsilon, 1 / \varepsilon$, and $\log (\varepsilon)$ on the temperature and electric field intensity have been calculated. The results of the calculations are illustrated in Figs. 1, 2, and 3. 


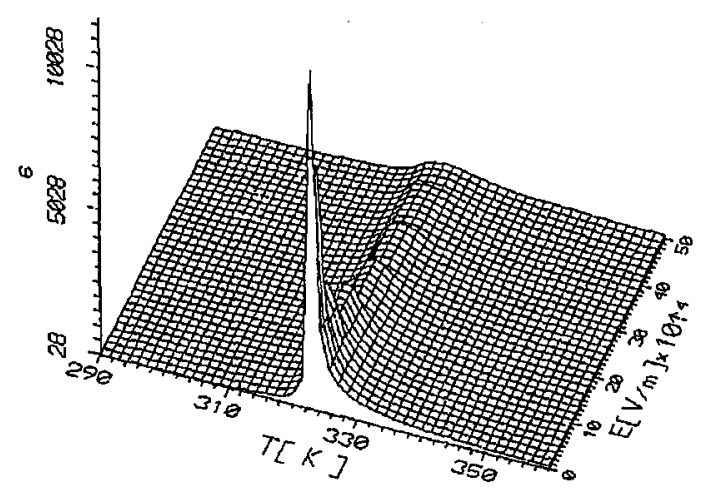

Fig. 1. Permittivity vs. temperature and electric field intensity calculated according to the thermodynamical theory.

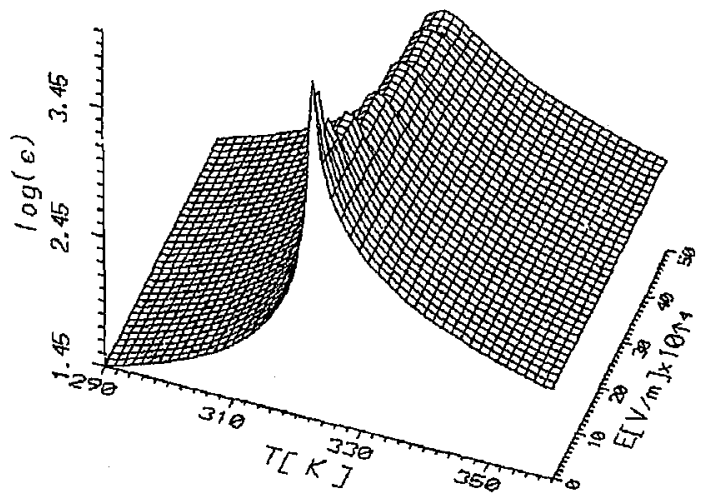

Fig. 2. $\log (\varepsilon)$ plotted as a function of temperature and electric field intensity calculated on the base of thermodynamical theory.

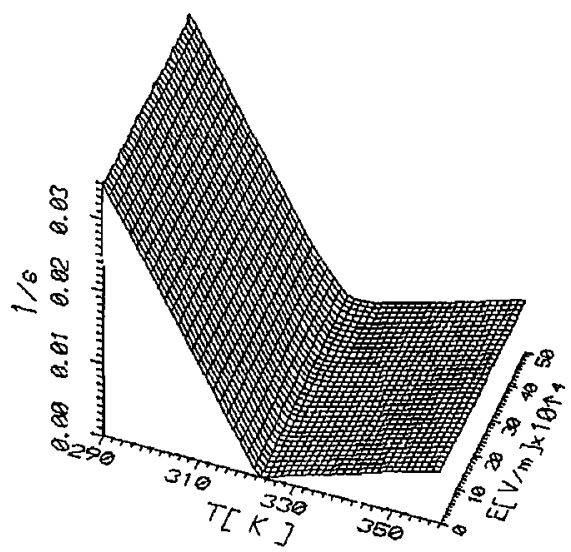

Fig. 3. Reciprocal of permittivity as a function of temperature and electric field intensity calculated on the base of thermodynamical theory. 


\section{Experimental}

The measurements of permittivity as a function of temperature and electric field intensity were done on a test stand, whose block diagram is shown in Fig. 4.

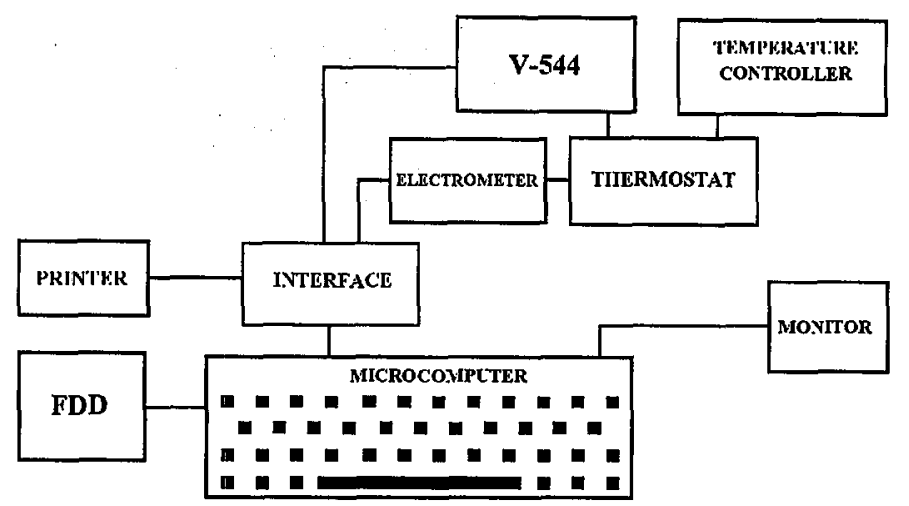

Fig. 4. Block diagram of the test stand used for measurement of permittivity vs. temperature and electric field.

The measurements were performed for the TGS crystals doped with the $\mathrm{Cr}^{3+}$ chromium ions at the concentration of $0.5 \%$ grown in the ferroelectric phase. The test samples for the measurements were cut in the disc shape with their flat surface perpendicular to the ferroelectric axis. On these surfaces, the silver electrodes were vacuum deposited. Thin copper wires were glued to electrodes using silver paste. These wires enabled a free suspension of the samples and also provided the electric connection. So prepared samples were located in the thermostat and held at a temperature of $360 \mathrm{~K}$ for 18 hours in order to achieve rejuvenation. Immediately after annealing, the measurements were performed. All the measurements were performed at a temperature change rate equal to $1 \mathrm{~K} / \mathrm{min}$.

\section{Results of the examination}

The measurements of the permittivity were performed for the TGS crystals doped with the $\mathrm{Cr}^{3+}$ chromium ions as a function of temperature at eight different values of electric field used to polarise the crystals. The experimental data were elaborated by means of the cringing method and presented in the 3-dimensional system using the $T-E$ grid.

The experimental results of the permittivity measurements are presented in Fig. 5. The obtained data confirmed the predicted ones resulting from the thermodynamical theory (Fig. 1). The electric field causes the change of the temperature dependence of the permittivity, and the influence of electric field is the bigger the closer the temperature approaches to a phase transition point. In all cases the electric field causes a diffusion of the phase transition and this diffusion increases with the rise of the value of the polarising field. The values of the permittivity at a temperature of $290 \mathrm{~K}$ obtained during the measurements are in good agreement with those obtained theoretically. The character of 




Fig. 5. Permittivity vs. temperature and electric field intensity for the TGS crystals doped with the $\mathrm{Cr}^{3+}$ chromium ions at the concentration of $0.5 \%$.

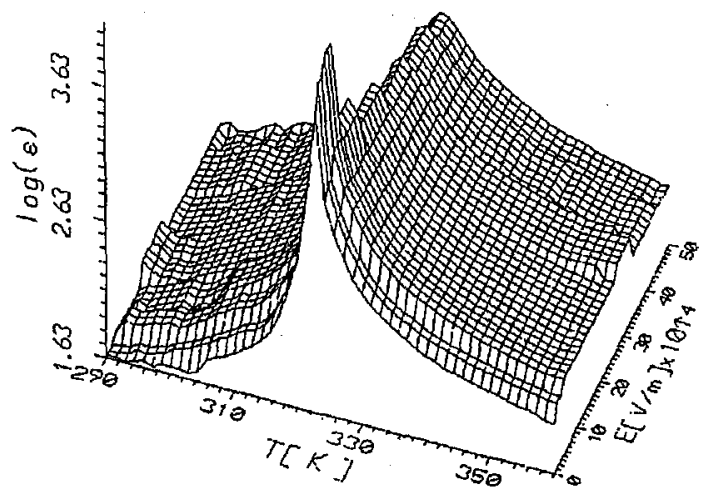

Fig. 6. Logarithm of the permittivity vs. temperature and electric field intensity for TGS crystals doped with $\mathrm{Cr}^{3+}$ ions at the concentration of $0.5 \%$.



Fig. 7. Reciprocal of the permittivity vs. temperature and electric field intensity for TGS crystals doped with $\mathrm{Cr}^{3+}$ ions at the concentration of $0.5 \%$. 
the changes of $\varepsilon$ with temperature and electric field intensity is the same for both, the theoretical and experimental dependences. The logarithm of the permittivity as a function of temperature and electric field polarising the crystals is plotted in Fig. 6. The reciprocal of the permittivity vs. temperature and electric field intensity for TGS crystals doped $\mathrm{Cr}^{3+}$ ions is shown in Fig. 7 .

It results from these measurements for the TGS crystals doped with the $\mathrm{Cr}^{3+}$ chromium ions that in the ferroelectric phase the permittivity does not depend on the value of electric field intensity at a temperature far from the phase transition point. On the other hand, the remarkable influence of electric field on $\varepsilon_{\max }$ (the maximum value of the permittivity near to the phase transition temperature) was found. The field polarising the crystal changes the region of peculiar variations of the permittivity. This region increases toward higher temperatures with an increase in electric field. After crossing the Curie point, the permittivity does not rapidly drop to the zero value but gradually diminishes (in such proportion as the paraelectric mass significantly exceeds the ferroelectric mass in the crystal). The value of $\varepsilon_{\max }$ decreases with the increase in electric field and simultaneously the half-width of the $\varepsilon=f(T)$ curve increases.

In Fig. 8 is shown the displacement of the Curie temperature as a function of the electric field polarising the crystal, where experiments were made during heating and cooling the crystal. There are also shown the results of Stankowska and Kiszkowski for a pure TGS crystal for which the dependence $\Delta T_{\mathrm{C}} \sim E^{2 / 3}$ is determinated [7].

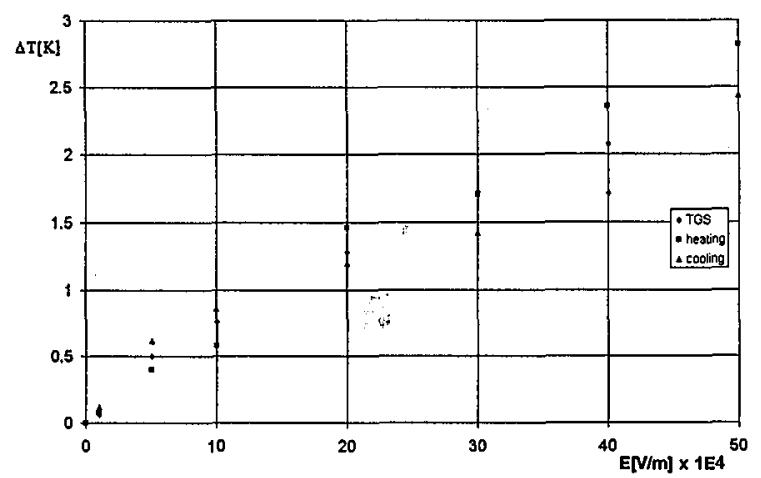

Fig. 8. The dependence of the displacement $\Delta T_{\mathrm{C}}$ from the polarising electric field for TGS crystals doped with $\mathrm{Cr}^{3+}$ ions at the concentration of $0.5 \%$ and undoped TGS according to [7].

A qualitative and quantitative character of the changes of permittivity as a function of temperature and electric field obtained from the experiment and calculated theoretically is in good agreement (with the exception of the relation $\varepsilon=f(T)$ at $E=0)$. 


\section{Discussion}

The measurements presented in this paper confirmed the relationship among permittivity, temperature and electric field polarising the crystal predicted on the grounds of the thermodynamical theory. This agreement is not only quantitative but also, with an acceptable accuracy, qualitative.

The electric field may distinguish some given direction of vibration of ions in the crystal lattice leading to the violation of the principle of equipartition of energy for the particular degrees of freedom. This effect increases with an increase in electric field intensity. This causes the distortion of the statistical distribution of vibration of ions in the lattice, which results in the dilution of the phase transition and in a shift of its temperature toward higher temperatures.

All $\varepsilon=f(T)$ dependences show the significant maxima at temperatures close to the phase transition point. These maxima are connected with the rapid disappearance of polarisation during the phase transition of the crystal.

These investigations did not give a convincing proof for the strict relationship between permittivity values in the ferroelectric phase at temperatures far from $T_{\mathrm{C}}$ and the electric field intensity. On the other hand, there is a remarkable relationship between these quantities in the close vicinity of the phase transition.

A certain proposal of explanation of this fact can be presented on the base of the results given in paper [8], which relate to the formation and decay of a weak bonding among chains formed by glicinium II, glicinium III and $\mathrm{SO}_{4}^{2-}$ groups bounded by glicinium I molecules at temperatures close to $T_{\mathrm{C}}$.

These bonds are responsible for the existence of dipole moments of elementary cells. Taking into account the statistical character of the formation and breaking of these bonds, it may be assumed that the probability of occurrence of these two phenomena is a different function of temperature. At each temperature from the critical range, the equilibrium is established between the formed and broken bonds and according to that the number of molecules contributing to this process is an ascending function of temperature approaching a maximum at a given temperature and then it decreases. The maximum value of this function and its half-width depend on physical properties of the crystal. Processes of the formation and breaking of the bonds lead to a change of the number of dipole moments in the crystal.

In the non-polarised crystal the breaking of the above-mentioned bonds begins in the ferroelectric phase in the vicinity of $T_{\mathrm{C}}$ whilst at $T_{\mathrm{C}}$ the kinetic energy of molecules is already large enough to break all the bonds, which leads to a disappearance of spontaneous polarisation. The above fact is reflected in the fast increase in the permittivity followed by its rapid drop to such value as in the paraelectric phase.

The electric field causes a temperature-induced dispersion of a number of molecules participating in the process of the formation and decay of bonds and also causes a shift of their maximum number towards higher temperatures with the increase in the electric field intensity. This fact is reflected in the behaviour of the permittivity. The increase in electric field leads to diminishing the maximum value 
of the permittivity and to an increase in the half-width of the function $\varepsilon=f(T)$ at $E=$ const.

We consider the crystal doped with chromium ions (see [2]) as a system of two phases. One of them consists of the non-disturbed crystal lattice and the other one is composed of the spherical regions disturbed by the $\mathrm{Cr}^{3+}$ impurities. Then the interaction of the electric field on the non-disturbed crystal is different. The electric field may cause the selective break dipoles away layers of the impurities. This is shown in Fig. 6 as an oscillation of the permittivity.

The chromium ion impurities cause the formation of the internal electric field, which is added to the field polarising the crystal. In the particular case, when the polarising electric field is directed in the opposite direction to the internal field, both fields are compensated. In this case many properties of the crystal, both electric and elastic, are similar to those which are present in a non-doped crystal.

\section{References}

[1] H. Wolniewicz, Mater., Sci. 17, 3 (1991).

[2] T. Krajewski, J. Dubik, E. Dubik, Fiz. Diel. Radiospektr. VIII/2, 189 (1976).

[3] S. Waplak, J. Stankowski, Acta Phys. Pol. 36, 71 (1969).

[4] R. Truell, Phys. Rev. 116, 890 (1959).

[5] C. Ying, R. Truell, J. Appl. Phys. 27, 1086 (1956).

[6] J. Malecki, Teoria Fal i Układów Akustycznych, PWN, Warszawa 1964 (in Polish).

[7] J. Stankowska, P. Kiszkowski, Fiz. Diel. Radiospektr. 3, 155 (1966).

[8] J. Stankowski, Ferroelectrics 28, 107 (1978). 